\title{
Klangmeditation in der onkologischen Rehabilitation: Pilotstudie zu einer rezeptiven Gruppenmusiktherapie mit dem Monochord*
}

\author{
Jens-Peter Rose Joachim Weis \\ Psychosoziale Abteilung, Klinik für Tumorbiologie, Freiburg i.Br., Deutschland
}

\author{
Schlüsselwörter \\ Musiktherapie - Onkologische Rehabilitation . \\ Entspannungsverfahren · Monochord · Evaluation
}

\section{Zusammenfassung}

Hintergrund: Musiktherapie kann als rezeptives Angebot in der psychoonkologischen Behandlung von Krebspatienten eine wichtige Rolle spielen. Wichtige Zielsetzungen liegen in der Unterstützung der Entspannung sowie der Krankheitsverarbeitung. Diese Studie befasst sich mit der Evaluation eines rezeptiven Gruppenmusiktherapieangebotes, der Klangmeditation, in einer onkologischen Rehabilitationsklinik. Im Zentrum der Untersuchung steht die Frage nach dem Erleben der Patienten während der Klangmeditation. Patienten und Methoden: Es handelt sich um eine 1-Gruppen-Prä-Post-Studie, in der Patienten vor und nach vier Klangmeditationssitzungen innerhalb von 3 Wochen untersucht wurden. Ein selbst entwickelter bipolarer Stimmungsfragebogen wurde von den Patienten vor und nach jeder Sitzung ausgefüllt und mit einer ANOVA mit Messwiederholung analysiert. Die Lebensqualität wurde mit dem EORTC QLQ-C30 Fragebogen vor der 1. Sitzung und am Ende des gesamten Angebots erfasst. Das Gesamterleben wurde über ein schriftliches, strukturiertes Kurzinterview erfasst und mit der Inhaltsanalyse nach Mayring qualitativ ausgewertet. Ergebnisse: Es wurden die Daten von 105 Patienten ausgewertet. Der Stimmungsfragebogen zeigte über den Verlauf der Sitzungen signifikante Veränderungen hin zu einem Gefühl von mehr Ausgeglichenheit, geminderter Nervosität und geminderter Erschöpfung. Die Klangmeditation bewirkte bei $75,6 \%$ der Patienten positive Körpersensationen, wie z.B. Schwereempfindungen der Extremitäten oder ein Durchströmtwerden von Wärme. Schlussfolgerungen: Trotz der Vorläufigkeit der Ergebnisse einer Pilotstudie ergeben sich Hinweise darauf, dass sich die Klangmeditation von Anfang an positiv auf die Entspannungsfähigkeit auswirkt. In weiteren Studien sollte geklärt werden, wie die verschiedenen Wirkelemente der Klangmeditation zusammenspielen und ob Wirknachweise auch in einem kontrolliert randomisierten Design möglich sind.

\author{
Key Words \\ Music therapy - Oncological rehabilitation . \\ Relaxation therapy · Monochord · Evaluation
}

\section{Summary}

Sound Meditation in Oncological Rehabilitation a Pilot Study of a Receptive Music Therapy Group Using the Monochord

Background: Music therapy can play an important role in psychooncology and promote relaxation or coping processes. This pilot study presents an evaluation of receptive music therapy performed in a group at an oncological rehabilitation hospital, with a focus on the experiences made by the patients. Patients and Methods: Single-group pre-post study on patients receiving at least 4 sessions of music therapy during a 3-week rehabilitation. A bipolar mood questionnaire was to be filled in by each participant before and after every session of music therapy. In addition, patients answered a questionnaire on quality of life (EORTC QLQ-C30) at the beginning and the end of the whole treatment. Data were analyzed by repeated-measures ANOVA. After the last session, the patients were to answer some open questions in writing. These texts were then analyzed by qualitative content analysis. Results: The data of 105 patients could be analyzed. The mood questionnaire revealed significant changes over the course of the intervention towards feeling more balanced, less nervous and less exhausted. 75.6\% of the patients reported positive body sensations such as feelings of heaviness or warmth of limbs during the intervention. Conclusions: The preliminary results of the pilot study suggest that sound meditation represents an effective relaxation therapy from the beginning of the treatment. In contrast to some other relaxation methods, sound meditation does not require regular exercise or practice to achieve positive effects. Further studies in terms of randomized controlled trials should investigate interactions of different elements of sound meditation with regard to its outcome.

*Das Projekt wurde gefördert durch die Hans-Georg-Laves-Stiftung und durchgeführt an der Klinik für Tumorbiologie in Freiburg.

\begin{tabular}{ll}
\hline KARGER & @ 2008 S. Karger GmbH, Freiburg \\
Fax +497614520714 & Accessible online at: \\
Information@Karger.de & www.karger.com/fok \\
www.karger.com &
\end{tabular}




\section{Einleitung}

In der Musiktherapie werden wie in keiner anderen künstlerischen Therapieform neben aktiven bzw. expressiven Methoden auch rezeptive Verfahren eingesetzt. Dies hängt unter anderem mit der Verarbeitung akustischer Signale durch unser Gehirn und der damit verbundenen Nähe zum emotionalen Erleben zusammen [1]. Gerade in der onkologischen Arbeit spielen rezeptive musiktherapeutische Interventionen als komplementärtherapeutisches Angebot eine wichtige Rolle. Wird in anderen Arbeitsfeldern der Musiktherapie, z.B. in der Psychiatrie, der Einsatz rezeptiver Elemente aus therapeutischen Gründen in Frage gestellt [2], bietet das Hören von Musik bzw. Klängen in der Psychoonkologie die Möglichkeit, therapeutische Ziele zu erreichen und die Patienten in ihrer Krankheitsverarbeitung zu unterstützen. Positive Effekte rezeptiver Musiktherapie können z.B. die Senkung des Muskeltonus, die Linderung von Schmerzen und die Förderung eines Entspannungs- oder Ruheerlebens sein [3].

Eine Krebserkrankung geht mit zahlreichen psychosozialen Belastungen einher, von der Verarbeitung massiver emotionaler Belastungen bis hin zur Auseinandersetzung mit existentiellen Themen wie dem eigenen Tod und Sterben. In der Literatur zur Musiktherapie in der Onkologie finden sich dementsprechend diverse Indikationsbereiche. So thematisieren kasuistisch angelegte Veröffentlichungen sowohl durch aktive als auch rezeptive Musiktherapie die Förderung von Entspannungszuständen [4], die Steigerung des Selbstwertgefühls [5, 6] oder die Reduzierung von Übelkeit und Schwindel [7] während der Chemotherapie. Auch der Abbau krankheitsbedingter Isolation [8] und die Anregung der Kommunikation unter den Patienten [9] bzw. mit der Familie [5, 6] wurden kasuistisch untersucht. Des Weiteren kann das aktive Musizieren den Ausdruck belastender Gefühle und eine damit einhergehende Verbesserung und Unterstützung des Copings [7, 10] fördern und ein Erleben von Hoffnung, Trost und Spiritualität [11] unterstützen. In der Begleitung Sterbender bieten sich rezeptive Verfahren der Musiktherapie an, da der Patient darüber auch in Phasen der Somnolenz oder Bewusstseinstrübung noch erreicht werden kann [12]. Quantitative Studien untersuchten unter anderem die unterstützende Wirkung rezeptiver Musik bei krankheitsbedingten Einschlafstörungen [13] und die Adjuvanz der medizinischen Therapie chronischer Krebsschmerzen [14-16]. Einige Studien [17, 18] fanden einen positiven Einfluss von Musik auf Ängste und depressive Gestimmtheit während der Verabreichung einer Chemotherapie [3].

Zur Klangwirkung und Verwendung des Monochords in der Musiktherapie finden sich vornehmlich im deutschsprachigen Raum Veröffentlichungen [19, 20]. Zeuch [21] betrachtet die Klangwirkung des Monochords aus phänomenologischer Sicht. Er geht davon aus, dass sich die durch den permanenten Gleichklang des Instrumentes entstehende Reizdeprivation, gekoppelt mit einer Reizüberflutung bedingt durch das sich ständig modulierende Obertonspektrum, für die Wirkung des Monochordklangs verantwortlich zeigt. Jungaberle et al. [22] berichten in einer qualitativen Untersuchung an Patienten von einer sehr guten Eignung des Monochords zur Herbeiführung körperlicher und geistiger Entspannung und intensiver Bewusstseinphänomene während des Hörens. Typische Reaktionen auf den Monochordklang konnten in der Untersuchung jedoch nicht aufgezeigt werden.

In einer Studie an Studenten untersuchte Hartogh [23] die Frage nach typischen Beurteilungen, Bildern und Assoziationen im Erleben des Monochordklangs und den Einfluss des Klangs auf die Befindlichkeit. Zum Einsatz kamen dabei ein für die Fragestellungen entwickeltes semantisches Differential und offen gestellte Fragen, die inhaltsanalytisch ausgewertet wurden. Die Studenten nannten als vorrangige Assoziation bei ihrem Hörerleben die Farbe Blau und die Elemente Luft und Wasser. Der Klang des Instrumentes wurde insgesamt als aggressionsabbauend, harmonisierend und entspannend charakterisiert. Hartogh betont den engen Zusammenhang von Monochordklang und Naturerleben, spricht sich im Weiteren aber gegen eine Interpretation dieses Erlebens der Probanden in Richtung einer pränatalen Regression aus, wie es an anderer Stelle diskutiert wurde [24].

\section{Beschreibung der Klangmeditation}

Bei der Klangmeditation in der Klinik für Tumorbiologie handelt es sich um ein komplexes, rezeptives, musiktherapeutisches Gruppenangebot. Im Zentrum des Verfahrens steht ein 30-saitiges Monochord, dessen permanenter Klang während einer Sitzung von Klangschalen, einem chinesischem TamtamGong und der Singstimme gerahmt wird. Das Monochord wird über die gesamte Klangphase hindurch gespielt. Zum rein klanglichen Angebot kommt unterstützend eine sprachliche Begleitung hinzu. Neben dem Monochord werden Gong, Klangschalen und Singstimme eingesetzt, um der Klangmeditation insgesamt einen dynamischen Verlauf zu verleihen.

Die Patienten liegen in der Regel auf dem Boden mit Matten, Decken und Kopfkissen und werden gebeten, eine angenehme Liegeposition zu wählen (meist Rückenlage). Wenn das Liegen auf dem Boden aus körperlichen Gründen nicht möglich ist, kann die Klangmeditation auch im Sitzen durchgeführt werden. Nach einer kurzen verbalen Einführung durch den Therapeuten mit Betonung auf der Position des Körpers und dem Strom der Atmung beginnt die Klangmeditation mit dem Erklingen des Gongs. Es folgen die Klangschalen, danach setzt das Monochord (Stimmung A, C und D) langsam ein und untermalt das Erleben der Teilnehmer in den restlichen 40 min. 10 min nach Einleiten der Klangphase durch den Gong kommt der Musiktherapeut mit einer vertiefenden, verbalen Entspannungsinstruktion hinzu, in welcher der Monochordklang mit positiven Assoziationen, wie etwa einem angenehmen Strömen der Klänge durch den Körper, verknüpft wird. 
Den Teilnehmern wird freigestellt, dieser stimmlichen Begleitung innerlich zu folgen. Im Anschluss folgt für 10 min eine Phase mit Gesang, zum Teil Obertongesang. Die Teilnehmenden können hier frei entscheiden, ob sie in den Gesang einstimmen wollen. Am Ende erfolgt der gemeinsame Ausklang mit einer verbalen Einleitung zur Reorientierung in den Musiktherapieraum. Den Teilnehmenden wird einige Minuten Zeit gelassen, um nach der teilweise intensiven Tiefenentspannung ins alltägliche Bewusstsein zurückzufinden. Abschließend moderiert der Musiktherapeut einen Gedankenaustausch zum Erleben der Teilnehmenden während der Ruhephase. Diese abschließende Phase der Reflexion dauerte bei einer durchschnittlichen Gruppengröße von 8 Personen üblicherweise 15-25 min. Die Intervention dauert insgesamt 90 min und wird in Gruppen von 4 bis maximal 8 Patienten durchgeführt. Pro Woche fanden für die Patienten in der Regel zwei Sitzungen in unregelmäßigen Abständen zwischen 2 und 5 Tagen statt.

\section{Fragestellung und Methodik der Studie}

Durch die Pilotstudie wurde das reguläre Angebot der Klangmeditation an der Klinik für Tumorbiologie von Dezember 1998 bis Januar 2002 evaluiert. Die Klangmeditation ist Teil eines umfassenden Rehabilitationsprogramms. Daher ist eine Ergebnisevaluation der Klangmeditation als Einzelmaßnahme nicht Gegenstand dieser Studie. Vielmehr konzentrierte sich diese Untersuchung auf die Identifikation von Prozessmerkmalen der Klangmeditation und unmittelbare Effekte auf die Aktualbefindlichkeit. Folgende Fragen standen im Mittelpunkt der Untersuchung:

1. Was kennzeichnet die Teilnehmer der Klangmeditation hinsichtlich psychosozialer und medizinischer Basisdaten und der Lebensqualität?

2. Wie beschreiben die Patienten ihr Erleben während einer Klangmeditation?

3. Welche Befindlichkeitsänderungen zeigen sich im Verlauf mehrerer musiktherapeutischer Sitzungen bei den Teilnehmern?

Es wurde ein prospektives 1-Gruppen-Prä-Post-Design gewählt, in dem nach einer Baseline-Erhebung (Lebensqualität, Erwartungen und Vorerfahrungen) vor und nach jeder Klangmeditationssitzung Fragebögen zur Befindlichkeit und zum subjektiven Erleben eingesetzt wurden. Nach der letzten Sitzung wurden ein abschließender Fragebogen zum Gesamteindruck, eine zweite Erhebung der Lebensqualität sowie ein kurzes, strukturiertes Interview in Freitextform durchgeführt.

In der Untersuchung wurde ausschließlich die Patientensicht erhoben. Ausgewählt wurden Fragebögen zur Erfassung der Aktualbefindlichkeit, zum subjektiven Erleben der Intervention und zur gesundheitsbezogenen Lebensqualität. Da in der musiktherapeutischen Forschung für die beiden erstgenannten Bereiche keine standardisierten Instrumente vorliegen, mussten die Verfahren speziell für diese Fragestellungen entwickelt werden. Es wurde ein aus acht Items bestehender Befindlichkeitsfragebogen konstruiert. Die Items bestanden aus einem 7-stufigen semantischen Differential und bezogen sich auf die Dimensionen Erschöpfung, Nervosität, Ausgeglichenheit, Ängstlichkeit, Hoffnung, Interesse, Zurückgezogenheit und Geborgenheit. Zum Abschluss des gesamten Therapieangebots wurden Daten zum Gesamterleben der Klangmeditation erfragt sowie drei offene Fragen gestellt, die im Freitext schriftlich zu beantworten waren. Darüber hinaus wurden zur Beschreibung der Teilnehmer medizinische und soziodemografische Merkmale sowie die Lebensqualität mithilfe des EORTC QLQ-C30 [25] zu Beginn und am Ende der Intervention erfasst.
Die für die Prozessanalyse der Klangmeditation selbst entwickelten Instrumente (Befindlichkeitsbogen, Erleben und Wahrnehmung der Klangmeditation, Abschlussbewertung) wurden in einer Testphase einer kleinen Anzahl von 5 Patienten vorgelegt, um ihre Verständlichkeit und Akzeptanz zu überprüfen. In der vorliegenden Studie sollten auch Daten zur Validierung dieser Fragebögen generiert werden.

Eingeschlossen wurden alle Patientinnen und Patienten, die im Rahmen einer mindestens 3-wöchigen Rehabilitation nach einer onkologischen Erkrankung in der Klinik für Tumorbiologie stationär aufgenommen waren und an der Klangmeditation teilnahmen. Die Teilnahme an der Studie war freiwillig. Um in die Auswertung einbezogen zu werden, sollten die Patienten mindestens vier Mal an der Klangmeditation teilgenommen haben. Die Patienten wurden vor der Studie schriftlich über die Befragung informiert und gaben ihr Einverständnis zur Teilnahme ebenfalls schriftlich («informed consent»). Nach Rücksprache mit dem Vorsitzenden der lokalen Ethikkommission war ein Ethikvotum nicht erforderlich, da die Intervention im Rahmen der klinischen Routine untersucht und keine für wissenschaftliche Zwecke eingeführte neue Intervention erprobt wurde.

Die Daten wurden über eine ANOVA mit Messwiederholung analysiert. Zudem wurden die Effektstärken zu den acht Parametern berechnet. Die Freitextantworten wurden mithilfe der qualitativen Inhaltsanalyse nach Mayring [26] analysiert.

\section{Ergebnisse}

\section{Stichprobenbeschreibung}

Es konnten die Daten von 105 der 134 befragten Patienten ausgewertet werden. Die anderen Patienten konnten nicht an mindestens vier Klangmeditationen teilnehmen. Dies lag vor allem an Überschneidungen mit anderen medizinisch-diagnostischen Terminen oder einer vorzeitigen Abreise.

Von den 105 auswertbaren Patienten war die überwiegende Anzahl weiblich (77,4\%). Das Alter lag im Durchschnitt bei 54,6 Jahren (Range 31-70). Die Mehrzahl war verheiratet (72,4\%), 12,3\% ledig, 8,4\% verwitwet und 6,7\% geschieden. Weiterhin erhielten $60 \%$ der untersuchten Patienten eine Erwerbsunfähigkeitsrente, $16,2 \%$ eine Altersrente und nur 6,8\% standen in einem Beschäftigtenverhältnis, davon 2,9\% Vollzeit, weitere $2,9 \%$ Halbzeit oder mehr und $1 \%$ weniger als Halbzeit. 17,1\% der Patienten waren als Hausfrau bzw. Hausmann tätig.

Über die Hälfte $(50,5 \%)$ der in die Studie eingeschlossenen Patienten war an Brustkrebs erkrankt, gefolgt von Krebserkrankungen des Verdauungstraktes (15\%); 66\% der Patienten waren tumorfrei bzw. in einer kompletten Remission, $7 \%$ in einer partiellen Remission und 7\% in einer progredienten Erkrankungssituation. Ein unklarer Krankheitsstatus, der weitere medizinisch-diagnostische Maßnahmen nach sich zog, lag bei $20 \%$ der Teilnehmer vor.

\section{Erfahrung mit Musik und Musiktherapie}

Nur $12 \%$ der Patienten hatte bereits Erfahrung mit Musiktherapie. Ein knappes Drittel (28\%) hatte bereits andere Entspannungsverfahren kennengelernt oder Erfahrungen mit Meditation (29\%). Bezüglich musikalischer Erfahrung gaben $38 \%$ an, ein Instrument zu spielen, während $62 \%$ musikalische 
Tab. 1. Patientenerwartungen an die Klangmeditation
Entspannung (geistige innere Ausgeglichenheit; Harmonisierung,

körperliche Entspannung, Besserung der Schlaflosigkeit )

Emotional (aufbauende Gefühle erleben; innere Unruhe und Ängste reduzieren,

Freude durch die Musik erfahren; gutes Gemeinschaftsgefühl)

Kognitiv (Ablenkung; positiv denken; Konzentrationsfähigkeit erhöhen)

Energie und Stabilität (Energie schöpfen; Sicherheit erleben)
64

16

14

6
Erfahrung ausschließlich durch das Hören von Musik gesammelt hatten.

Auf die offen gestellte Frage nach den Erwartungen an die Klangmeditation antworteten 92 Patienten. Aus diesen Antworten ließen sich 151 unterschiedliche Nennungen identifizieren. Eine Analyse der Antworten mit der qualitativen Inhaltsanalyse nach Mayring ergab vier Antwortkategorien (Tab. 1).

\section{Validierung des Befindlichkeitsfragebogens}

Der Befindlichkeitsfragebogen wurde zunächst einer Faktorenanalyse unterzogen, um die Dimensionalität zu überprüfen. Hierfür wurde ein Item (Nr. 7 Zurückgezogenheit) umgepolt. Mit der Hauptachsenmethode konnte ein Generalfaktor abgebildet werden, der rund $31 \%$ der Varianz erklärt und als Befindlichkeit bezeichnet werden kann. Aufgrund dieser Ergebnisse lässt sich ein Summenwert bilden, der den nachfolgenden Analysen zugrunde gelegt wurde.

Zur weiteren Überprüfung wurde die interne Konsistenz (Cronbachs $\alpha$ ) für die jeweiligen Messzeitpunkte errechnet. Für die Messungen vor den Klangmeditationssitzungen ergaben sich Werte zwischen 0,73 und 0,87, für die Messungen nach den Sitzungen Werte zwischen 0,77 und 0,84, was für eine zufriedenstellende bis gute interne Konsistenz der Skala spricht.

Der Summenwert des Befindlichkeitsfragebogens korrelierte gering mit den Skalen körperliche, soziale, kognitive sowie Rollenfunktion des EORTC QLQ-C30 und der globalen Lebensqualität. Alle Symptomskalen des EORTC QLQ-C30, wie etwa zu Schmerz oder Schlaflosigkeit, korrelierten erwartungsgemäß niedrig negativ mit dem Summenwert des Befindlichkeitsfragebogens. Nur die Skala «Emotional Functioning» über alle vier Sitzungen korrelierte signifikant $(p \leq 0,005)$ mit dem Summenwert des Befindlichkeitsfragebogens ( $\mathrm{r}$ zwischen $0,28$ und 0,44$)$. Die absoluten Korrelationswerte variieren geringfügig über die verschiedenen Messzeitpunkte. In Bezug auf die Konstruktvalidität weisen die Ergebnisse insgesamt darauf hin, dass der Befindlichkeitsfragebogen im Wesentlichen die emotionale Befindlichkeit erfasst.

\section{Ergebnisse des Befindlichkeitsfragebogens im Verlauf}

Der Befindlichkeitsfragebogen war das zentrale Instrument zur Erfassung der Veränderung der Befindlichkeit in jeder Klangmeditationssitzung. Die Analyse wurde mittels einer einfaktoriellen ANOVA mit Messwiederholung durchgeführt.
Die vier Parameter Müdigkeit / Frische, Ruhe, Ausgeglichenheit und Geborgenheit zeigten in der Messung direkt nach der Intervention (post) eine signifikante Veränderung hin zum Positiven, verglichen mit der Befindlichkeit vor der Klangmeditation (prä). Diese Besserung war nach jeder Sitzung vergleichbar ausgeprägt und blieb über den Verlauf der vier Sitzungen konstant. Jede Klangmeditationssitzung bewirkte in diesen Erlebensbereichen positive Veränderungen. Eine signifikante Steigerung der Befindlichkeiten über den Verlauf der vier Sitzungen konnte jedoch nicht festgestellt werden.

Die Parameter Ängstlichkeit, Hoffnung, Interesse und Zurückgezogenheit zeigten hingegen keine signifikante Veränderung im Prä-Post-Vergleich der einzelnen Sitzungen. Das Interesse wurde von den Patienten mit einem Mittelwert von $5,9$ und der geringsten Standardabweichung ( $\mathrm{SD}=1,27)$ der acht Items insgesamt hoch eingestuft. In Abbildung 1 sind die signifikanten Verläufe grafisch dargestellt.

Die Berechnung der Effektstärken diente wie bereits erwähnt nicht der Effektivitätsbeurteilung, sondern der Abschätzung möglicher zu erwartender Effekte. Sie wurden berechnet über die gemittelten Prä- minus Post-Werte der vier Sitzungen. Die Effektstärken des Befindlichkeitsfragebogens waren für Ruhe und Ausgeglichenheit gemäß Cohen mittelstark und für Geborgenheit, Müdigkeit / Frische, Ängstlichkeit und Hoffnung klein (Tab. 2). Die Effekte der Parameter Interesse und Zurückgezogenheit waren nicht bedeutsam.

\section{Erleben während der Klangmeditation}

Die Teilnehmer wurden nach jeder Sitzung nach ihrer individuellen Klangwahrnehmung befragt. Es wurden vier Items in Form eines semantischen Differentials zum Erleben der Klangintensität vorgegeben, die auf einer Skala von 1 bis $7 \mathrm{zu}$ bewerten waren.

Aus jeder Sitzung konnten die Daten von 103 Teilnehmern ausgewertet werden (ANOVA mit Messwiederholung). Die Klänge (Item: Ich höre die Klänge immer (1) - gar nicht (7)) wurden nahezu permanent gehört (Range 2,48-2,85), was der Verlauf über die vier Sitzungen untermauert. Die Stärke der Klänge (stark (1) - schwach (7)) wurde zwischen 3,72 und 3,79 auf der 7-stufigen Skala verortet. Auch die Distanz (nah (1) fern (7)) zu den gehörten Klängen wurde nahezu identisch mit durchschnittlich 3,65 bewertet. Nur die erlebte Lautstärke lag mit durchschnittlich 4,37 etwas über der Skalenmitte von vier. Es ergaben sich keine signifikanten Veränderungen über den Verlauf der vier Sitzungen. 


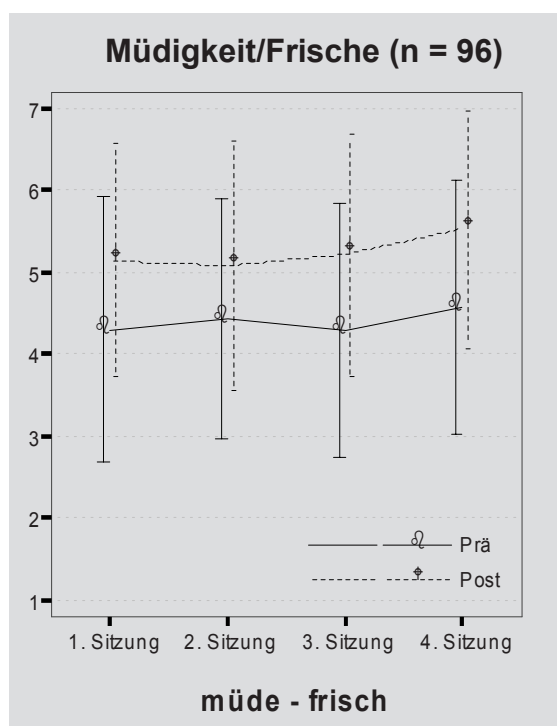

Ausgeglichenheit $(n=100)$

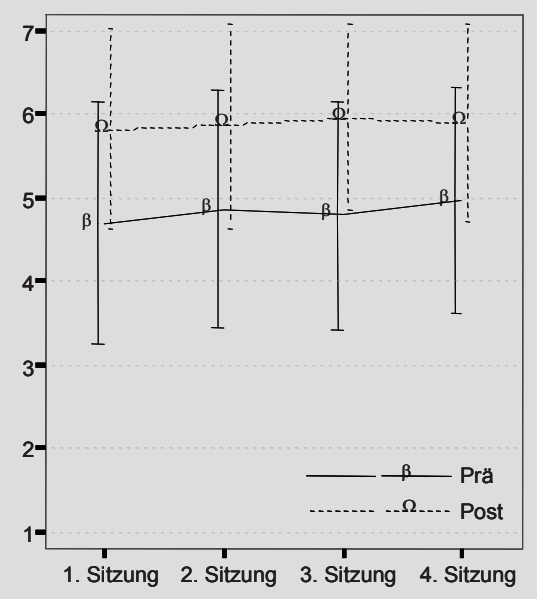

unausgeglichen - ausgeglichen

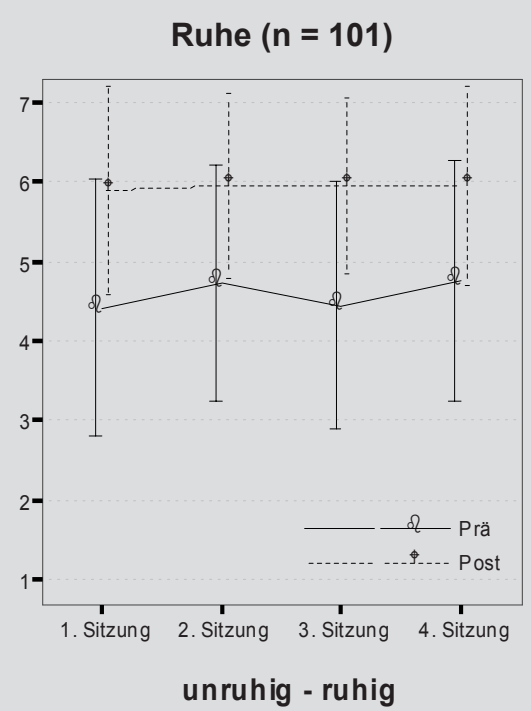

Geborgenheit $(n=101)$

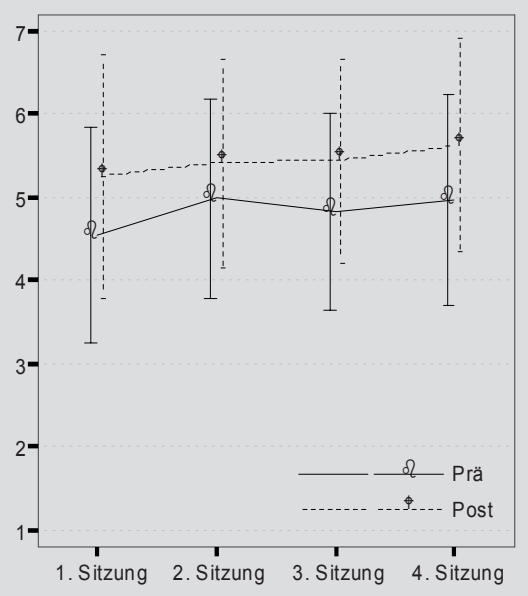

geborgen: gar nicht - sehr stark nach jeder Klangmeditationssitzung.

Tab. 2. Effektstärken (d) der Parameter des Befindlichkeitsfragebogens ${ }^{\mathrm{a}}$

\begin{tabular}{llll}
\hline & & $\mathrm{d}$ \\
\hline 1 & Müdigkeit / Frische & müde (1) - frisch (7) & 0,350 \\
2 & Ruhe & unruhig (1) - ruhig (7) & 0,655 \\
3 & Ausgeglichenheit & Unausgeglichen (1) - ausgeglichen (7) & 0,605 \\
4 & Ängstlichkeit & ängstlich (1) - angstfrei (7) & 0,297 \\
5 & Hoffnung & hoffnungslos (1) - hoffnungsvoll (7) & 0,208 \\
6 & Interesse & Interessiert: gar nicht (1) - sehr stark (7) & 0,001 \\
7 & Zurückgezogenheit & zurückgezogen: gar nicht (1) - sehr stark (7) & 0,015 \\
8 & Geborgenheit & geborgen: gar nicht (1) - sehr stark (7) & 0,371 \\
\hline
\end{tabular}

aPrä-Wert - Post-Wert der gemittelten Sitzungen.

Ein weiterer Fragenkomplex erfasste mit 5 Items das durch die Klänge induzierte Erleben. Hierzu wurde für verschiedene Erlebensweisen global gefragt: «Wie wirken die Klänge?» Auf einer 7-stufigen, bipolaren Skala (gar nicht (1) - sehr (7)) konnte jedes Item eingestuft werden (Abb. 2).

Am höchsten wurde die beruhigende Wirkung der Klänge mit einem Gesamtmittelwert von 5,59 über die vier Sitzungen eingeschätzt. Die tiefenentspannende Wirkung der Klänge («Die Klänge tragen fort») wurde mit $\mathrm{M}=5,27$ ebenfalls hoch eingestuft. Generell wurden die Klänge als sehr angenehm erlebt ( «Die Klänge sind mir unangenehm»; $M=2,05$ ). Die Einschätzung der ermüdenden Wirkung der Klänge lag mit $\mathrm{M}=3,58$ in 


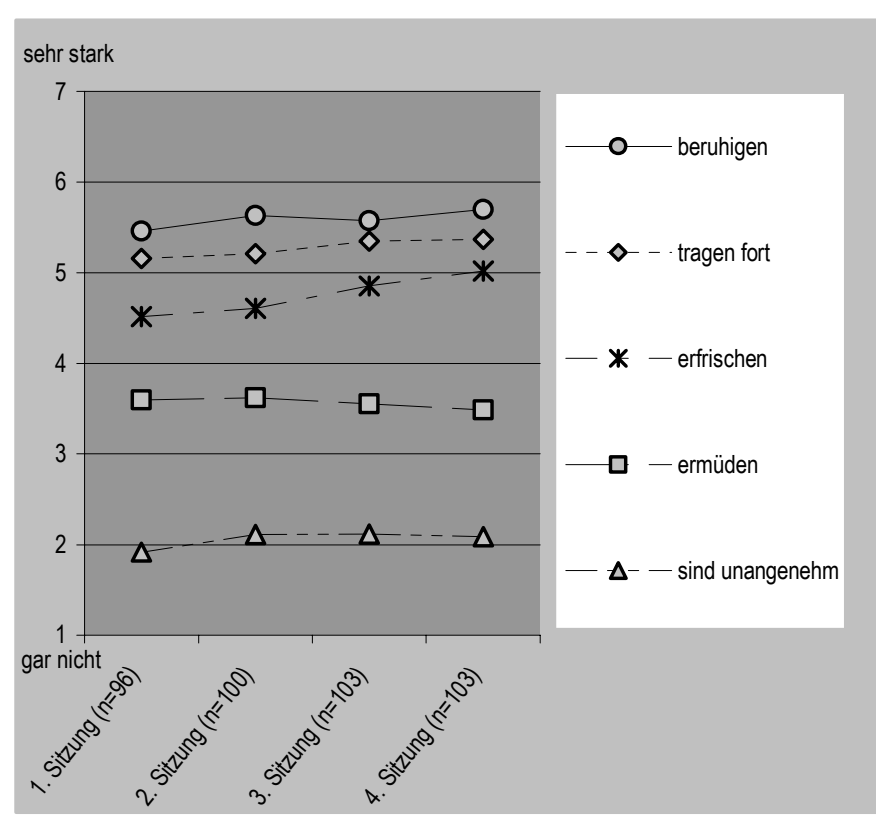

Abb. 2. Wirkung der Klänge I (Wie wirken die Klänge? - Einstufung von $1-7)$.

der Mitte der beiden Pole. Die erfrischende Wirkung der Klänge wurde mit $M=4,69$ deutlich in der oberen Hälfte der Skala eingestuft. Über den Verlauf der vier Sitzungen (ANOVA mit Messwiederholung) zeigte jedoch nur dieses Item eine signifikant positive Veränderung $(\mathrm{F}=3,16 ; \mathrm{p}=$ $0,025)$. Die Klänge hatten somit im Verlauf der vier Sitzungen eine zunehmend erfrischende Wirkung auf die Teilnehmer. Die Ausprägung aller anderen Items blieb weitgehend unverändert.

Des Weiteren wurden die Teilnehmer angehalten, im Anschluss an jede Klangmeditation 8 Ja-Nein-Fragen zu ihrem Erleben zu beantworten. Von Interesse waren die Sinnesrepräsentationen «Innere Bilder», «Farben», «Körper-», «Geruchs-» und «Geschmacksempfindungen» sowie das Erleben von «Erinnerungen», «Emotionen» und der «Einfluss auf Schmerzen (falls vorhanden)». Hier konnten die Antwortfragebögen von 95 Patienten ausgewertet werden (Abb. 3).

Bei 79,8\% der Patienten wurden während einer Klangmeditation Erinnerungen hervorgerufen. Körperempfindungen wurden mit 78,3\% von ähnlich vielen Patienten erlebt. Im Verlauf der vier Sitzungen nahm der Anteil der Patienten, die Emotionen, innere Bilder und Farben erlebten, deutlich zu. Dies zeigt der Chi-Quadrat-Test, der hier zur Orientierung dient, im Vergleich der ersten und vierten Sitzung, bei dem alle drei Erlebensmodalitäten schwach signifikant $(\mathrm{p}<0,05)$ werden. Geruchs- und Geschmacksempfindungen wurden durchschnittlich von 7,3 bzw. 4,5\% der Patienten erlebt; sie spielten damit eine untergeordnete Rolle und hatten wenig Einfluss auf das Erleben während einer Klangmeditation. Nur rund $30 \%$ der Befragten gaben an, dass die Klänge ihr Schmerzerleben beeinflussten.

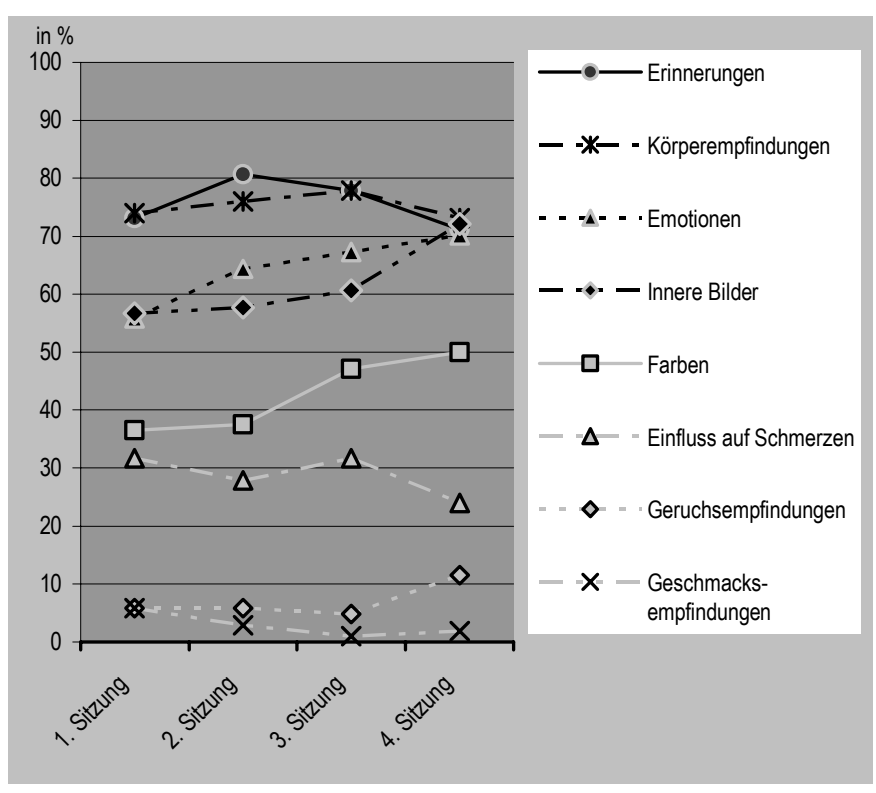

Abb. 3. Wirkung der Klänge II (Was lösen die Klänge aus? - Häufigkeit von Ja-Antworten).

\section{Lebensqualität}

Zur Charakterisierung der Teilnehmer wurde der EORTC QLQ-C30 eingesetzt. Das Profil der Lebensqualität zeigt, dass hinsichtlich der Funktionswerte vor allem emotionale und soziale Einschränkungen vorlagen. Geringere Einschränkungen lagen bei Rollenfunktion, kognitiven und körperlichen Funktionen vor. Die Teilnehmer der Klangmeditation zeigten somit erwartungsgemäß ein Profil, das auf psychosozialen Behandlungsbedarf hinweist. Bei den Symptomskalen zeigten sich die größten Probleme in den Bereichen Erschöpfung (Fatigue) und Schlafstörungen.

Im Sinne eines allgemeinen Rehabilitationseffektes am Ende der Reha-Maßnahme zeigten sich signifikante Verbesserungen nur in den beiden Skalen emotionale Funktion und globale Lebensqualität $(p \leq 0,001)$. Die Veränderungen sind jedoch, wie eingangs erwähnt, nicht ursächlich auf die Klangmeditation zurückzuführen (Abb. 4).

\section{Qualitative Auswertung der offenen Abschlussfragen}

Was hat besonders gut getan? Auf diese Abschlussfrage gaben 89 der befragten 105 Patienten schriftliche Antworten. Diese wurden anhand der qualitativen Inhaltsanalyse nach Mayring [26] in 151 Einzelnennungen unterteilt und zu 5 übergeordneten Antwortkategorien zusammengefasst (Tab. 3). Am häufigsten wurde mit 48,9\% ein Erleben von seelischem Wohlbefinden im Sinne von Entspannung, einer veränderten Einstellung zum Leben, veränderten Emotionen oder Entspannung als neuem Verarbeitungsmuster beim Gedanken an die Krebserkrankung genannt. Etwa ein Drittel der Nennungen bezog sich direkt auf die eingesetzten Klänge und die Musik als besonders wichtiges Erfahrungselement. Die Atmosphäre 
Abb. 4. Lebensqualität vor und nach dem Therapieangebot $(* *$ emotional functioning: $\mathrm{p}=0,001 ; * *$ global health status $\mathrm{p}<0,001)$.

Tab. 3. Antwortkategorien auf die Frage: «Was hat besonders gut getan?» $(\mathrm{n}=98$ Patienten $)$

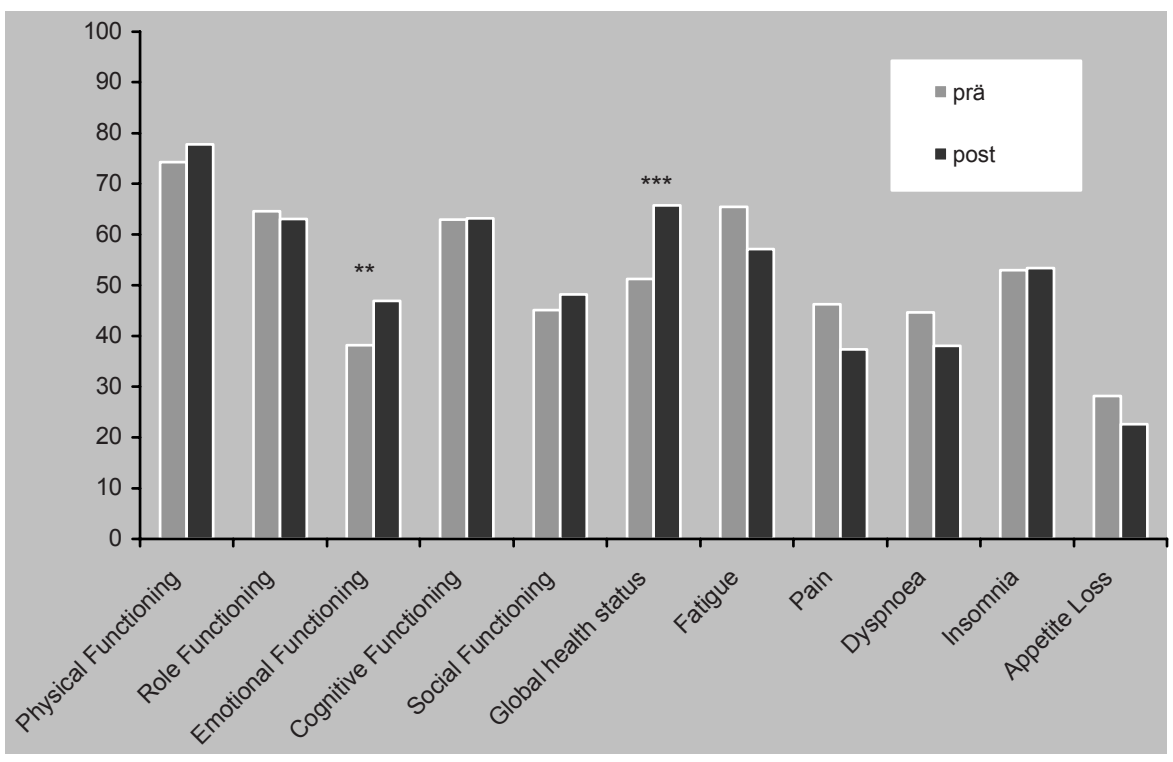

\begin{tabular}{lc}
\hline & $\%^{\mathrm{a}}$ \\
\hline 1. Das Erleben psychischen Wohlbefindens & 48,9 \\
Entspannung (44) & 13,5 \\
Neue Einstellung zum Leben (11) & 11,5 \\
Veränderte Emotionen (9) & 9,3 \\
Entspannende Gedanken als neues Verarbeitungsmuster (8) & 8,3 \\
Umgang mit der Erkrankung (4) & 4,2 \\
Spirituelle Erfahrung (2) & 2,1 \\
\hline 2. Die Klänge / die Musik (44) & 30,2 \\
\hline 3. Die Atmosphäre / das Setting & 10,4 \\
Die stimmliche Begleitung (15) & 4,2 \\
Der anschließende Gedankenaustausch in der Gruppe (4) & 3,1 \\
Die Atmosphäre (3) & 3,1 \\
Die explizite Erlaubnis, nichts tun zu müssen (3) & 7,3 \\
\hline 4. Das Erleben körperlicher Veränderungen & 3,1 \\
Leichtigkeit des Körpers (3) & 3,1 \\
Körperliche Entspannung (3) & 1,1 \\
Schmerzfreiheit (1) & 3,1 \\
\hline 5. Die Kombination einer sprachlichen Anleitung mit Live-Musik (3) & \\
\hline a Von 151 Nennungen. &
\end{tabular}

und das Setting wurden bei 10\% der Nennungen erwähnt. Einige Patienten erlebten während der vier Klangmeditationssitzungen Veränderungen des körperlichen Erlebens als besonders unterstützend. Dreimal wurde die Kombination von sprachlicher Anleitung und Musik als besonders hilfreich beschrieben.

Was war Ihr tiefstes Erlebnis mit der Klangmeditation? Auf diese Frage antworteten 91 Patienten. Die Antworten wurden in insgesamt 126 Einzelnennungen unterteilt, aus denen $9 \mathrm{Ka}-$ tegorien gebildet werden konnten (Tab. 4). Der Schwerpunkt der Patientenantworten lag dabei deutlich auf Erlebensweisen, die sich häufig im Zuge von Tranceerfahrungen und Tie- fenentspannung zeigen. Im Rahmen der Klangmeditation handelte es sich dabei zunächst um ein stark imaginativ geprägtes Erleben $(23,0 \%)$, ein verändertes körperliches oder psychisches Selbsterleben (20,6\%) einhergehend mit intensiven psychophysischen Entspannungsphänomenen (16,7\%). Zudem trug das Hören der Klänge zur Ablenkung von belastenden Gedanken bei (10,4\%). Des Weiteren wurden das Auftauchen von im biografischen Sinne alten, teilweise vergessen geglaubten Erinnerungen (9,5\%), intensiven Emotionen $(8,7 \%)$ und klanglich assoziierten Körpersensationen, wie etwa ein Strömen oder Fließen der Klänge durch den Körper, $(8,7 \%)$ genannt. 
Tab. 4. Antwortkategorien auf die Frage: «Was war das tiefste Erlebnis mit der Klangmeditation?» $(\mathrm{n}=91$ Patienten $)$

\begin{tabular}{lc}
\hline & $\%^{\mathrm{a}}$ \\
\hline 1. Imaginationen, Bilder und Farben & 23,0 \\
2. Verändertes Wachbewusstsein: Gefühl der Körperlevitation, vom Körper & \\
losgelöst sein, Eintritt in eine andere Welt & 20,6 \\
3. Körperliche und psychische Entspannung: tiefe Entspannung, innere Ruhe & 16,7 \\
4. Ablenkung der Gedanken durch Hören von Klängen: Intensive Hörerfahrung, & \\
$\quad$ Abstand zu Problemen, Verschwinden aller Gedanken & 10,4 \\
5. Starke Erinnerungen & 9,5 \\
6. Intensive Gefühle: Gefühle der Freude, weinen können & 8,7 \\
7. Körpersensationen: den Klang körperlich spüren, Wärme, Leichtigkeit & 8,7 \\
8. Neue Energie & 1,6 \\
9. Gruppenerleben: offen über ein persönliches Problem vor der Gruppe & 0,8 \\
zu sprechen & \\
\hline aVon 126 Nennungen. & \\
\hline
\end{tabular}

\section{Diskussion}

Mit der vorliegenden Pilotstudie wurde erstmalig eine systematische Prozessevaluation einer komplexen musiktherapeutischen Entspannungsintervention («Klangmeditation») durchgeführt. Ziel war es, erste deskriptive und prozessuale Merkmale dieser neuen Musiktherapieform zu beschreiben und zu analysieren, um Hypothesen für weiterführende Fragestellungen zu generieren.

Im Hinblick auf die Merkmale der Teilnehmer zeigen die Ergebnisse, dass das Diagnosespektrum breit gefächert war und vor allem weibliche Patientinnen dieses Angebot wahrnahmen. Auch bei anderen psychosozialen Angeboten haben wir einen höheren Anteil an Frauen, was mit einer größeren Offenheit sowie einer rollenspezifischen Krankheitsverarbeitung erklärt werden kann. Hinsichtlich der Lebensqualität zeichneten sich die Teilnehmer als vorrangig psychosozial behandlungsbedürftig aus. Offensichtlich spiegelt die Selbstzuweisung über den subjektiv wahrgenommenen Bedarf wie auch die Zuweisung über die psychologische Sprechstunde dies erfolgreich wider.

Weiterhin zeigen die Ergebnisse, dass die Klangmeditation mit dem Monochord den Krebspatienten einen schnellen und intensiven Zugang zu einem tiefen, psychophysischen Entspannungserleben ermöglichte.

Fasst man die Ergebnisse zusammen, so lässt sich sagen, dass die Klangmeditation als rezeptives Gruppenmusiktherapieangebot für erwachsene Krebspatienten die Möglichkeit bietet, tiefe Entspannung einhergehend mit einem starken imaginativen Erleben und deutlichen Veränderungen im körperlichen Selbsterleben zu erreichen. Besonders der Parameter Frische bzw. Erfrischung spielte bei der Befindlichkeitsmessung vor und nach jeder Sitzung und in Bezug auf die Klangwirkung nach jeder Sitzung eine wichtige Rolle. Die Befindlichkeitsparameter Erschöpfung, Nervosität, seelische Ausgeglichenheit und Geborgenheit zeigten im Prä-post-Vergleich jeder Klangmeditation ebenfalls positive signifikante Veränderungen.
Jede Klangmeditationssitzung bewirkte bei diesen Parametern eine Besserung der subjektiven Befindlichkeit, ohne dass über den Verlauf der Sitzungen eine wesentliche Steigerung erfolgte. Somit konnte keine wesentliche Verbesserung der Befindlichkeit über den Verlauf von vier Klangmeditationssitzungen festgestellt werden. Lediglich die Wirkung der Klänge wurde über den Verlauf der Sitzungen als zunehmend erfrischend beschrieben.

Die Befragungen nach den einzelnen Klangmeditationen zeigten hinsichtlich des Erlebens der Klänge eine zunehmende Wahrnehmung emotionaler Anteile über den Verlauf der Sitzungen. Auch die Wahrnehmung innerer Bilder und Farben nahm signifikant zu. In der Regel wurden klangassoziierte Körperempfindungen, innere Bilder, Emotionen und Erinnerungen von drei Vierteln der teilnehmenden Patienten erlebt. Dies spricht für einen Übungseffekt, der möglicherweise noch verbesserbar wäre. Die Mindestteilnahme an vier Sitzungen wurde eher aus praktischen Gründen festgesetzt als aufgrund einer therapeutischen Indikation. In der stationären Rehabilitation ist in der verfügbaren Zeit nur eine begrenzte Therapiedichte in Abhängigkeit vom sonstigen Programm möglich. Dennoch sollten zukünftige Studien die Abstände zwischen den Klangmeditationen sowie die Zeitpunkte bedenken. Während unserer Studie wurde die Klangmeditation entweder zweimal vormittags oder einmal nachmittags angeboten. In den anschließenden Nachbesprechungen berichteten viele Teilnehmende von Problemen, während der morgendlichen Klangmeditation zur Ruhe zu kommen, während sie am späteren Nachtmittag keine Probleme hatten.

Die Daten des Lebensqualitätsfragebogens EORTC QLQC30, der primär zur Beschreibung der Teilnehmer eingesetzt wurde, zeigen zumindest in zwei Funktionssubskalen (globaler Gesundheitsstatus, emotionale Funktion) signifikante Veränderungen am Ende der Intervention. Aufgrund des Fehlens einer Kontrollgruppe sowie zahlreicher Einflüsse anderer Interventionen im Rahmen der stationären Rehabilitation können diese Ergebnisse nicht als Effekt der Klangmeditation 
interpretiert werden. Dennoch legen die Ergebnisse es nahe, in zukünftigen kontrollierten Studien auch die Lebensqualität als Zielparameter einzusetzen.

Insgesamt zeigen die Befunde, dass die Klangmeditation eine komplexe Intervention ist, die vor allem Prozesse der Tiefenentspannung bewirkt, zugleich jedoch auch eine Reihe damit assoziierter Wahrnehmungsinhalte und intrapsychischer Erlebensweisen initiieren kann. Eine der zentralen Fragen der Studie, ob sich die Befindlichkeit im Verlauf von vier Klangmeditationssitzungen ändert, konnte nicht bestätigt werden. Lediglich die Wirkung der Klänge wurde als zunehmend erfrischend beschrieben.

Dies bestätigte auch die qualitative Auswertung der offenen Fragen, wonach fast die Hälfte der Patienten ein durch die Klangmeditation induziertes seelisches Wohlbefinden erreichte und die Klangerfahrung als außerordentlich wohltuend beschrieb. Dabei zählten innere Bilder, Imaginationen, Wahrnehmungsveränderungen und klangassoziierte Körperempfindungen zu den intensivsten Erlebensweisen der Patienten. Als zentrales Erleben wurde hier die psychophysische Entspan- nungsintensität benannt. Es bestätigten sich die Inhalte einer primär rezeptiven Intervention, der sich ein breites Anwendungsfeld eröffnet. Spezifische Wirkungen in Richtung auf eine Schmerzbeeinflussung, die in der Literatur breit diskutiert werden [13-15], lassen sich durch die vorliegenden Ergebnisse nicht bestätigen, lagen jedoch auch nicht im Fokus der Studie. Die Ergebnisse aus dem Bereich der Wahrnehmungslenkung lassen jedoch erwarten, dass sich die Klangmeditation auch bei Schmerzproblemen einsetzen ließe. Dies zu untersuchen, ist nachfolgenden Studien vorbehalten und bedarf eines spezifischen Studienansatzes, in dem der Grad der erlebten Schmerzen detailliert erfasst wird.

Weitere Untersuchungen sollten eine Kontrollgruppe einbeziehen und standardisierte, validierte Messinstrumente verwenden. Dennoch weisen die vorliegenden Daten auf eine vielversprechende Wirkung und Intensität des musiktherapeutisch-rezeptiven Entspannungsverfahrens mit dem Monochord hin und lassen für zukünftige Studien gute Ergebnisse erwarten.

\section{Literatur}

1 Spitzer M: Musik im Kopf. Stuttgart, Schattauer, 2002.

2 Moser J: Die Wirkung von Musikinstrumenten in psychotherapeutischen Prozessen; in Müller L, Petzold HG (ed): Musiktherapie in der klinischen Arbeit. Stuttgart, Fischer, 1997, pp 186-207.

3 Rose J-P, Naar K, Weis J: Musiktherapie in der Onkologie. Konzepte und Stand der Forschung. Psychother Psych Med 2004;54:457-470.

4 Bailey LM: The effects of live music versus taperecorded music on hospitalized cancer patients. Music Ther 1983;3:17-28.

5 Munro S, Mount B: Music therapy in palliative care. Can Med Assoc J 1978;119:1029-1034.

6 Bailey LM: The use of songs in music therapy with cancer patients and their families. Music Ther 1984;4:165-171.

7 Porchet-Munro S: Music therapy; in Doyle D, Hanks G, MacDonald N (eds): Oxford Textbook of Palliative Medicine. Oxford, University Press, 1993, pp 555-559.

8 Porchet-Munro S: The supportive role of music. The active and passive roles music can play in palliative care. Eur J Palliat Care 1995;2:77-80.

9 Bunt L, Marston-Wyld J: Where words fail music takes over. A collaborative study by a music therapist and a counselor in the context of cancer care. Music Ther Perspect 1995;13:46-50.
10 Bold S: The effects of music therapy on motivation, psychological well-being, physical comfort, and exercise endurance of bone marrow transplant patients. J Music Ther 1996;33:164-188.

11 Weber S: Trost durch Musik; in Aldridge D (ed): Kairos IV: Beiträge zur Musiktherapie in der Medizin. Bern, Huber, 2000, pp 74-83.

12 Munro S: Musiktherapie bei Sterbenden. Stuttgart, Fischer, 1986.

13 Reinhardt U: Untersuchungen zur Synchronisation von Herzfrequenz und musikalischem Rhythmus im Rahmen einer Entspannungstherapie bei Patienten mit tumorbedingten Schmerzen. Forsch Komplementärmed 1999;6:135-141.

14 Zimmerman L, Pozehl B, Duncan K, Schmitz R: Effects of music in patients who had chronic cancer pain. West J Nurs Res 1989;11:298-309.

15 Beck SL: The therapeutic use of music for cancerrelated pain. Oncol Nurs Forum 1991;18:1327-1337.

16 Gallagher LM, Lagman L, Walsh D, Davis MP, LeGrand SB: The clinical effects of music therapy in palliative medicine. Supp Care Cancer 2006;14: 859-866.

17 Sabo CE, Michael SR: The Influence of personal message with music on anxiety and side effects associated with chemotherapy. Canc Nurs 1996;19: 283-289.

18 Standley JM: Clinical applications of music and chemotherapy: The effects on nausea and emesis. Music Ther Perspect 1992;10:27-35.
19 Dosch J, Timmermann T: Das Buch vom Monochord. Wiesbaden, Reichert, 2005.

20 Timmermann T: Das Monochord. Eine Wiederentdeckung. Musikther Umsch 1989;10:308-320

21 Zeuch A: Schöpfer eigener Wirklichkeiten. Die Klangmeditation mit dem Monochord. Musik Tanz Kunsttherapie 1999;10:175-185.

22 Jungaberle H, Altieri P, Gerloff E, Kurze K, Verres $\mathrm{R}$ : Töne strömen ein. Beiträge zur Musikpsychologie des Monochords. Eine qualitative Studie. Musikther Umsch 2003;24:319-332.

23 Hartogh T: Die Rezeption monotonaler Klänge. Musik Tanz Kunsttherapie 2001;12:111-119.

24 Strobel W: Klang - Trance - Heilung. Die archetypische Welt der Klänge in der Psychotherapie. Musikther Umsch 1988;9:119-139.

25 Aaronson NK, Ahmedzai S, Bergman B, Bullinger M, Cull A, Duez NJ, Filiberti A, Flechtner H, Fleishman SB, de Haes JCJM, Kaasa S, Klee MC, Osoba D, Razavi D, Rofe PB, Schraub S, Sneeuw KCA, Sullivan M, Takeda F, The European Organisation for Research and Treatment of Cancer QLQ-C30: A quality-of-life instrument for use in international clinical trials in oncology. J Natl Cancer Inst 1993;85:365-376.

26 Mayring P: Qualitative Inhaltsanalyse. Grundlagen und Techniken, ed. 7, Weinheim, Deutscher Studien Verlag, 2000. 